\title{
Operation cost reduction in unit commitment problem using improved quantum binary PSO algorithm
}

\author{
Ali Nasser Hussain, Ali Abduladheem Ismail \\ Department of Electrical Power Engineering Techniques, Electrical Engineering Technical College, \\ Middle Technical University, Baghdad, Iraq
}

\begin{tabular}{l} 
Article Info \\
\hline Article history: \\
Received May 31,2019 \\
Revised Oct 10, 2019 \\
Accepted Oct 18, 2019 \\
\hline Keywords: \\
Hybrid algorithm \\
Improved QBPSO algorithm \\
Particle swarm optimization \\
Quantum computing \\
Unit commitment
\end{tabular}

\begin{abstract}
Unit Commitment (UC) is a nonlinear mixed integer-programming problem. UC used to minimize the operational cost of the generation units in a power system by scheduling some of generators in $\mathrm{ON}$ state and the other generators in OFF state according to the total power outputs of generation units, load demand and the constraints of power system. This paper proposes an Improved Quantum Binary Particle Swarm Optimization (IQBPSO) algorithm. The tests have been made on a 10-units simulation system and the results show the improvement in an operation cost reduction after using the proposed algorithm compared with the ordinary Quantum Binary Particle Swarm Optimization (QBPSO) algorithm
\end{abstract}

Copyright () 2020 Institute of Advanced Engineering and Science. All rights reserved.

\section{Corresponding Author:}

Ali Nasser Hussain,

Department of Electrical Power Engineering Techniques,

Electrical Engineering Technical College,

Middle Technical University, Baghdad, Iraq.

Email: alinasser1974@yahoo.com

\section{INTRODUCTION}

Unit commitment is a hard problem that to be optimized in a power system in order to minimize the total operation cost of generators for a specified time horizon according to the load demand satisfying the power outputs of generators and system constraints [1]. UC has to make a decision to properly operate the generators to get a lower cost by making some generators $\mathrm{ON}$ and the others OFF according to the demand and these generators must be economically dispatched. UC problem is NP-hard problem and it can be presented as mixed integer nonlinear optimization problem. As the number of the generators grow up, the solution will take a longer time because the combinations 0-1 that for each hour in the time horizon will grow exponentially. Two types of constraints must be satisfied in the unit commitment problem solution, the first one is related to the system such as the transmission constraints and the power reserve constrains in case of increase the demand or the outage of a generator from the system and the other types of constraints are related to the generators such as ramp-up limit, ramp-down limit, minimum time up and minimum time down [2].

Different ideas have been developed to solve UC problem. The solution methods of the UC problem can be separated into two kinds, the first one is known as deterministic solution techniques such as Priority List (PL) [3]; Dynamic Programming (DP) [4]; Lagrangian Relaxation (LR) [5]; second order cone programming [6]; Mixed Integer Programming (MIP) [7]; Mixed Integer Linear Programming (MIP) [8] and Branch and Bound (BB) [9]. The other solution method is known as stochastic approaches and they were successful in UC problem solution and as an example for these methods Genetic Algorithm (GA) [10]; Evolutionary Programming (EP) [11]; Simulated Annealing (SA) [12]; Particle Swarm Optimization (PSO) [13]; Quantum Evolutionary Algorithm (QEA) [14]; Ant Colony Search Algorithm (ACSA) [15]; 
Differential Evolution approach (DE) [16]; Artificial Neural Networks (ANN) [17]; Tabu Search (TS) [18]; Eagle strategy based crow search algorithm (ES-CSA) [19] and Grey Wolf Optimizer (GWO) [20]. In this paper, the proposed algorithm is Improved Quantum Binary Particle Swarm Optimization (IQBPSO) algorithm which the product of the hybridization of Binary Particle Swarm Optimization (BPSO) algorithm and the Quantum Computing because the BPSO may fail to find the global minimum therefore this improvement has been made to achieve the better solution.

\section{PROBLEM FORMULATION}

The objective function of UC problem was formulated to minimize the total operation cost over the time horizon [1]. This minmization may be done by selecting the combination of the generation units that satisfies all the constraints of the power system and the generation unit itself and these combinations $0-1$ that represented the status of each generator ON/OFF. The formulation of cost function for the UC problem that must be minimized by the sum of the starting cost of the generators and operational cost for each unit over a specefied period of time and it can be expressed by the following equation:

$$
\mathrm{F}=\sum_{\mathrm{k}=1}^{\mathrm{T}} \sum_{\mathrm{g}=1}^{\mathrm{N}}\left[\mathrm{f}_{\mathrm{gk}}\left(\mathrm{P}_{\mathrm{gk}}\right)+\mathrm{STC}_{\mathrm{gk}}\left(1-\mathrm{U}_{\mathrm{g}(\mathrm{k}-1)}\right] \mathrm{U}_{\mathrm{gk}}\right.
$$

where $\mathrm{f}_{\mathrm{gk}}$ is the fuel cost function, $\mathrm{U}_{\mathrm{gk}}$ is the state of unit $\mathrm{g}$ which can be 0 or 1 at the hour $\mathrm{k}, \mathrm{N}$ is the number of generation units, $\mathrm{T}$ is the time horizon, $\mathrm{g}$ is the index of the unit, $\mathrm{k}$ is the index of time, $\mathrm{P}_{\mathrm{gk}}$ is the power delivered from the unit $\mathrm{g}$ at the hour $\mathrm{k}$ and $\mathrm{STC}_{\mathrm{gk}}$ is the start-up cost of the unit $\mathrm{g}$ at the hour $\mathrm{k}$.

The fuel cost function is calculated as follows

$$
f_{g k}\left(P_{g k}\right)=c_{g}\left(P_{g k}\right)^{2}+b_{g}\left(P_{g k}\right)+a_{g}
$$

where $c_{g}, b_{g}, a_{g}$ are the fuel cost coeficients and the start-up are represented by the following equation:

$$
\mathrm{STC}_{\mathrm{gk}}=\left\{\begin{array}{l}
\mathrm{HSC}_{\mathrm{g}} \text { if } \mathrm{MDT}_{\mathrm{g}} \leq \mathrm{T}_{\mathrm{g}}^{\mathrm{off}} \leq \mathrm{MDT}_{\mathrm{g}}+\mathrm{CSH}_{\mathrm{g}} \\
\mathrm{CSC}_{\mathrm{g}} \text { if } \mathrm{T}_{\mathrm{g}}^{\text {off }}>\mathrm{MDT}_{\mathrm{g}}+\mathrm{CSH}_{\mathrm{g}}
\end{array}\right.
$$

where $\left(\mathrm{HSC}_{\mathrm{g}}, \mathrm{CSC}_{\mathrm{g}}\right)$ are the hot and cold start-up cost of the unit $\mathrm{g}$; $\mathrm{CSH}_{\mathrm{g}}$ is the cold start hours for the unit $\mathrm{g}$; $\left(\mathrm{MUT}_{\mathrm{g}}, \mathrm{MDT}_{\mathrm{g}}\right)$ are the minimum up and downtime of the unit $\mathrm{g}$ and $\left(\mathrm{T}_{\mathrm{g}}^{\mathrm{on}}, \mathrm{T}_{\mathrm{g}}^{\text {off }}\right)$ are the time of the unit $\mathrm{g}$ is continuously $\mathrm{ON}$ or $\mathrm{OFF}$

The objective function of UC problem is resterercted by some constraints and these constraints are the system constraints and the generation unit constraints [1].

1. The demand must be supplied by the generators at each hour.

$$
\sum_{\mathrm{g}=1}^{\mathrm{N}} \mathrm{P}_{\mathrm{gk}} \mathrm{U}_{\mathrm{gk}}=\mathrm{D}_{\mathrm{k}}
$$

2. The constraint of spinning reserve in case of increase the demand or loss generator unit from the group.

$$
\sum_{\mathrm{g}=1}^{\mathrm{N}} \mathrm{P}_{\mathrm{g}}^{\max } \mathrm{U}_{\mathrm{gk}} \geq \mathrm{D}_{\mathrm{k}}+\mathrm{R}_{\mathrm{k}}
$$

3. The generator can produce power in the range between the maximum and minimum values.

$$
\mathrm{P}_{\mathrm{g}}^{\max } \geq \mathrm{P}_{\mathrm{gk}} \geq \mathrm{P}_{\mathrm{g}}^{\min }
$$

4. The generation unit must be operated at least for a time equals to the minimum up time.

$$
\mathrm{T}_{\mathrm{g}}^{\mathrm{on}} \geq \mathrm{MUT}_{\mathrm{g}}
$$

5. The generation unit must be shut-down or in the OFF state at least for a time equals to the minimum downtime.

$$
\mathrm{T}_{\mathrm{g}}^{\text {off }} \geq \mathrm{MDT}_{\mathrm{g}}
$$

where $D_{k}$ is the load demand of the system at the hour $k ; R_{k}$ is the spinning reserve of the system at the hour $\mathrm{k}$ and $\left(\mathrm{P}_{\mathrm{g}}^{\max }, \mathrm{P}_{\mathrm{g}}^{\min }\right)$ are the maximum and minimum power that can be supplied from the unit $\mathrm{g}$. 


\section{PARTICLE SWARM OPTIMIZATION AGORITHM}

Particle Swarm Optimization (PSO) is introduced firstly by James Kennedy and Russell Eberhart in 1995 [21]. PSO algorithm is a heuristic optimization method based on the parallel experience of the individuals to search for the optimum solution. The PSO particles spread in a search space D of the problem and each of them has a position vector $\boldsymbol{X}$ and speed vector $\boldsymbol{V}$ [21]. In this algorithm, the particles are guided using the personal experience for each particle which is known as Pbest and the overall or the global experience among all particles that termed as Gbest. Then, the velocity and location of each particle in the population are modified by using the calculation of the current particle velocity and the distance from Pbest location and Gbest location [22]. Furthermore, the experience can be accelerated by two set of the acceleration factors, $\left(c_{1}, c_{2}\right)$ are the cognitive and asocial acceleration constant factors respectively; $\left(\varphi_{1}, \varphi_{2}\right)$ are two random numbers generated between $[0,1]$. The movement is also can be controlled by multiplying it by inertia factor that lies in the range of $\left[\omega_{\max }, \omega_{\min }\right]$ and the typical range is $\omega_{\max }=0.9$ to $\omega_{\min }=0.4$. The velocity update is described by the following equation:

$$
V^{m+1}=\omega V^{m}+c_{1} \varphi_{1}\left(\text { Pbest }_{i}^{m}-X_{i}^{m}\right)+c_{2} \varphi_{2}\left(\text { Gbest }^{m}-X_{i}^{m}\right)
$$

where $\mathrm{m}$ is the current itration. The position of the particles can be updated as follows equation:

$$
X_{i}^{m+1}=X_{i}^{m}+V_{i}^{m+1}
$$

the inertia factor is represented by the following equation:

$$
\omega=\omega_{\max }-\frac{\left(\omega_{\max }-\omega_{\min }\right)}{\text { iter }_{\max }} \times m
$$

where $\left(\omega_{\max }, \omega_{\min }\right)$ are the initial and final weights, iter $_{\max }$ is the maximum iteration number and $m$ is the current iteration number. The binary version of the PSO (BPSO) has been presented by James Kennedy and Russell Eberhart to be used in discrete spaces [23]. The update proces of the position for the particles can be achieved by using a new variable known as Sigmoid Limiting Transformation and can be written as

$$
S\left(V_{i j}^{m+1}\right)=\frac{1}{1-\exp \left(V_{i j}^{m+1}\right)}
$$

By using the sigmoid function, the position update of the particle in the binary version of a PSO algorithm is done as the following equation

$$
X_{i j}^{m+1}=\left\{\begin{array}{l}
1 \text { if } r_{i j}<S\left(V_{i j}^{m+1}\right) \\
0 \text { otherwise }
\end{array}\right.
$$

where $r_{i j}$ is a random number distributed uniformly between $[0,1]$.

\section{HYBRIDIZATION OF QUANTUM COMPUTING WITH BPSO ALGORITHM}

The quantum bit is known as the smallest unit of information that store in the quantum computer [24]. The quantum bit can be in two states, the first state is 0 and the second is 1 . These states may be written as $|0\rangle$ and $|1\rangle$ and the quantum bit state can be reproduced as follows:

$$
|\Psi\rangle=\alpha|0\rangle+\beta|1\rangle
$$

where $\alpha$ and $\beta$ are two complex numbers that identifies the probability amplitude of the relative conditions. The state of the quantum bit can be normalized to unity to guarantee that $|\alpha|^{2}+|\beta|^{2}=1$. Quantum gates have been used to change the state of the quantum bit and for examples of these gates, NOT gate, Hadamard gate and rotation gate [25]. The novel QEA has been proposed by Kim and Han as-[24]. This QEA is inspired from the quantum-computing concept so the quantum bit has been designed to get the binary solutions. The quantum bit is defined by pair of numbers which are $\alpha$ and $\beta$ and the quantum bit can be formulated as a string of

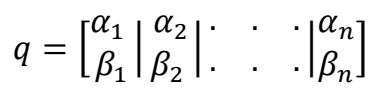


where $\left|\alpha_{j}\right|^{2}+\left|\beta_{j}\right|^{2}=1$ and $\mathrm{j}=1,2 \ldots \ldots \mathrm{n}$.

The rotation gate can be used as a variance factor to update the individual of the quantum bit and the rotation gate is represented by the following equation:

$$
U\left(\Delta \theta_{j}\right)=\left[\begin{array}{cc}
\cos \left(\Delta \theta_{j}\right) & -\sin \left(\Delta \theta_{j}\right) \\
\sin \left(\Delta \theta_{j}\right) & \cos \left(\Delta \theta_{j}\right)
\end{array}\right]
$$

where $\Delta \theta_{j}$ is the jth quantum bit rotation angle that goes to 0 or 1 state. A lookup table as shown in Table 1 is used to determine the value of $\Delta \theta_{j}$ and adjusted as $\theta_{1}=0, \theta_{2}=0, \theta_{3}=0.01 \pi, \theta_{4}=0, \theta_{5}=-0.01 \pi$, $\theta_{6}=0, \theta_{7}=0, \theta_{8}=0$ and $\mathrm{B}$ is the best solution where $\mathrm{B}=\left(b_{1}, b_{2}, b_{3}, \ldots \ldots, b_{n}\right)$ as described in reference [24].

Table 1. Lookup table to determine rotation angle

\begin{tabular}{cccc}
\hline$x_{j}$ & $b_{j}$ & Fitness $(\mathrm{X}) \geq$ Fitness $(\mathrm{B})$ & $\Delta \theta_{\mathrm{j}}$ \\
\hline 0 & 0 & False & $\theta_{1}$ \\
0 & 0 & True & $\theta_{2}$ \\
0 & 1 & False & $\theta_{3}$ \\
0 & 1 & True & $\theta_{4}$ \\
1 & 0 & False & $\theta_{5}$ \\
1 & 0 & True & $\theta_{6}$ \\
1 & 1 & False & $\theta_{7}$ \\
1 & 1 & True & $\theta_{8}$ \\
\hline
\end{tabular}

A new BPSO inspired by quantum computing which is known as Quantum Binary Particle Swarm Optimization (QBPSO) [26]. Each element in the particle has a state of 1 or 0 according to the probability of $|\alpha|^{2}+|\beta|^{2}=1$. The QBPSO proposes a new way to update the velocity of each particle by the use of Quantum Computing. The inertia factors $\left(\omega_{\max }, \omega_{\min }\right)$ and the acceleration factors $\left(c_{1}, c_{2}\right)$ are omitted in the QBPSO and replaced by the rotation angle. The update process of the position vector can be done by using the probability $|\beta|^{2}$ that has been stored in the $i$ th quantum bit individual $\left(q_{i}\right)$. Therefore, the $j$ th element of the $i$ th particle takes a value of 1 or 0 as in the following equation [26]:

$$
X_{i j}^{m+1}=\left\{\begin{array}{l}
1 \text { if } r_{i j}<\left|\beta_{i j}\right|^{2} \\
0 \text { otherwise }
\end{array}\right.
$$

The rotation angle can be determined by using the current position Pbest and the global position Gbest of the swarm as in the following equation:

$$
\Delta \theta_{i j}=\theta \times\left\{\gamma_{1 i} \times\left(x_{i j}^{P}-x_{i j}\right)+\gamma_{2 i} \times\left(x_{j}^{G}-x_{i j}\right)\right.
$$

where $\theta$ is the rotation angle magnitude and $\left(\gamma_{1 i}, \gamma_{2 i}\right)$ can be found by a comparison among the fitness of the current position of the particle $i$, the fitness of the best position Pbest and the fitness of the global position Gbest respectively as in equations (19) and (20):

$$
\begin{aligned}
& \gamma_{1 i}=\left\{\begin{array}{l}
0 \text { if } \text { Fitness of }\left(X_{i}\right) \geq \text { Fitness }\left(\text { Pbest }_{i}\right) \\
1 \text { otherwise }
\end{array}\right. \\
& \gamma_{2 i}=\left\{\begin{array}{l}
0 \text { if } \text { Fitness of }\left(X_{i}\right) \geq \text { Fitness }\left(\text { Gbest }_{i}\right) \\
1 \text { otherwise }
\end{array}\right.
\end{aligned}
$$

The magnitude of the rotation is decreased monotonously from a maximum value $\theta_{\max }$ to a minimum value $\theta_{\min }$ along the iteration by the following equation:

$$
\theta=\theta_{\max }-\frac{\theta_{\max }-\theta_{\min }}{\text { iter }_{\max }} \times m
$$




\section{IMPROVED QBPSO ALGORITHM}

The QBPSO algorithm may fail in finding the optimum value of the solution, therefor; an improvement is made on the QBPSO to get the better solution. The improvement on QBPSO is to search for the fitness in the personal best Pbest for the first half of the iterations and after finding it the fitness in the global best Gbest will be searched in the second half of the iterations. This improvement can be expressed as the following equations:

$$
\begin{aligned}
& \gamma_{1 i}=\left\{\begin{array}{l}
0 \text { if } m \geq\left(\text { iter }_{\text {max }} / 2\right) \\
1 \text { otherwise }
\end{array}\right. \\
& \gamma_{2 i}=\left\{\begin{array}{l}
1 \text { if } m \geq\left(\text { iter }_{\text {max }} / 2\right) \\
0 \text { otherwise }
\end{array}\right.
\end{aligned}
$$

and the rotation angle is updated as in $(18,21)$

\section{RESULTS AND ANALYSIS}

The simulation is implemented using MATLAB program version (R17b) for test system consist of 10 generation units over a time horizon of 24 hours [26]. The values of $\theta_{\min }$ and $\theta_{\max }$ are chosen equal to $0.05 \pi$ and $0.1 \pi$ respectively. The number of population and iterations are 50 and 26 respectively. Table 2 lists the generation of each unit. The total operation cost that has been obtained by the IQBPSO is 563938.4\$ where the total fuel cost of the generation units is 559848.4 $\$$ and the sum of the startup cost for all the units is $4090 \$$. The results showed minimum cost has been achieved from using the IQBPSO compared with the ordinary QBPSO which produces a total cost of 563977\$ as in reference [26].

\begin{tabular}{|c|c|c|c|c|c|c|c|c|c|c|c|}
\hline hour & Unit1 & Unit 2 & Unit 3 & Unit 4 & Unit 5 & Unit 6 & Unit 7 & Unit 8 & Unit 9 & Unit 10 & Demand MW \\
\hline 1 & 455 & 245 & 0 & 0 & 0 & 0 & 0 & 0 & 0 & 0 & 700 \\
\hline 2 & 455 & 295 & 0 & 0 & 0 & 0 & 0 & 0 & 0 & 0 & 750 \\
\hline 3 & 455 & 370 & 0 & 0 & 25 & 0 & 0 & 0 & 0 & 0 & 850 \\
\hline 5 & 455 & 390 & 0 & 130 & 25 & 0 & 0 & 0 & 0 & 0 & 1000 \\
\hline 6 & 455 & 360 & 130 & 130 & 25 & 0 & 0 & 0 & 0 & 0 & 1100 \\
\hline 7 & 455 & 410 & 130 & 130 & 25 & 0 & 0 & 0 & 0 & 0 & 1150 \\
\hline 10 & 455 & 455 & 130 & 130 & 162 & 33 & 25 & 10 & 0 & 0 & 1400 \\
\hline 11 & 455 & 455 & 130 & 130 & 162 & 72.8 & 25 & 10.2 & 10 & 0 & 1450 \\
\hline 12 & 455 & 455 & 130 & 130 & 162 & 80 & 25 & 43 & 10 & 10 & 1500 \\
\hline 13 & 455 & 455 & 130 & 130 & 162 & 33 & 25 & 10 & 0 & 0 & 1400 \\
\hline 14 & 455 & 455 & 130 & 130 & 85 & 20 & 25 & 0 & 0 & 0 & 1300 \\
\hline 19 & 455 & 455 & 130 & 130 & 30 & 0 & 0 & 0 & 0 & 0 & 1200 \\
\hline 20 & 455 & 455 & 130 & 130 & 162 & 33 & 25 & 10 & 0 & 0 & 1400 \\
\hline 21 & 455 & 455 & 130 & 130 & 85 & 20 & 25 & 0 & 0 & 0 & 1300 \\
\hline 22 & 455 & 455 & 0 & 0 & 145 & 20 & 25 & 0 & 0 & 0 & 1100 \\
\hline 23 & 455 & 425 & 0 & 0 & 0 & 20 & 0 & 0 & 0 & 0 & 900 \\
\hline 24 & 454.79 & 345.21 & 0 & 0 & 0 & 0 & 0 & 0 & 0 & 0 & 800 \\
\hline
\end{tabular}

Table 2. Results of UC problem in the simulation system

\section{CONCLUSION}

The unit commitment problem is a hard optimization process that the power system has to be deal with it to get a minimum operation cost. This paper proposes an improved quantum binary particle swarm optimization (IQBPSO) algorithm to solve the unit commitment problem. The algorithm has been tested on 10 unit simulation system during 24-hour time horizon and a minimized operational cost has been achieved. 


\section{REFERENCES}

[1] Wood and B. Wollenberg, "Power generation, operation, and control". New York: J. Wiley \& Sons, 1996.

[2] Hobbs, et al., The Next Generation of Electric Power Unit Commitment Models. Boston, MA: Springer US, 2002.

[3] R. M. Burns and C. A. Gibson, "Optimization of priority lists for a unit commitment program," presented at the IEEE Power Engineering Society Summer Meeting, 1975, Paper A, 75 453-1.

[4] W. L. Snyder, et al., "Dynamic programming approach to unit commitment," IEEE Trans. Power Syst., vol. 2, no. 2, pp. 339-350, May 1987.

[5] F. Zhuang and F. D. Galiana, "Toward a more rigorous and practical unit commitment by Lagrangian relaxation," IEEE Trans. Power Syst., vol. 3, no. 2, pp. 763-770, May 1988.

[6] Yuan, Xiaohui, et al., "Second-order cone programming for solving unit commitment strategy of thermal generators". Energy Conversion and Management. vol. 76, pp. 20-25, 2013. 10.1016/j.enconman.2013.07.019.

[7] A. I. Cohen and M. Yoshimura, "A branch-and-bound algorithm for unit commitment," IEEE Trans. Power App. Syst., vol. PAS-102, pp. 444-451, Feb. 1983.

[8] M. Shaaban, H. Zeynal, and K. Nor, "MILP-based short-term thermal unit commitment and hydrothermal scheduling including cascaded reservoirs and fuel constraints," International Journal of Electrical and Computer Engineering (IJECE), vol. 9, no. 4, pp. 2732-2742, Aug. 2019.

[9] J. A. Muckstadt and R. C. Wilson, "An application of mixed-integer programming duality to scheduling thermal generating systems,” IEEE Trans. Power App. Syst., vol. PAS-87, pp. 1968-1978, 1968.

[10] K. S. Swarup and S. Yamashiro, "Unit commitment solution methodology using genetic algorithm," IEEE Trans. Power Syst., vol. 17, no. 1, pp. 87-91, Feb. 2002.

[11] H. Chen and X. Wang, "Cooperative coevolutionary algorithm for unit commitment," IEEE Trans. Power Syst., vol. 16, no. 1, pp. 128-133, Feb. 2002.

[12] D. N. Simopoulos, et al.,, "Unit commitment by an enhanced simulated annealing algorithm," IEEE Trans. Power Syst., vol. 21, no. 1, pp. 68-76, Feb. 2006.

[13] B. Zhao, et al., "An Improved Particle Swarm Optimization Algorithm for Unit Commitment," Elect. Power Energy Syst., Vol. 28, No. 7, pp. 482-490, 2006.

[14] T. W. Lau, et al., "Quantum-inspired Evolutionary Algorithm Approach for Unit Commitment," IEEE Trans. Power Syst., Vol. 24, No. 3, pp. 1503-1512, Aug. 2009.

[15] PS. Sishaj, et al., "An Ant Colony System Approach for Unit Commitment Problem," Int J Electr Power Energy Syst, Vol. 28, No. 5, pp. 315-23, 2006.

[16] X. Yuan, et al., "Application of Enhanced Discrete Differential Evolution Approach to Unit Commitment Problem," Energy Convers Manage, Vol. 50, pp. 2449-2456, 2009. 28

[17] H. Sasaki, et al., "A Solution Method of Unit Commitment by Artificial Neural Networks," IEEE Trans Power Syst, Vol. 7, No. 3, pp. 974-81, 1992.

[18] A. Borghetti, et al., "Lagrangian Relaxation and Tabu Search Approaches for Unit Commitment Problem," IEEE porto power tech conference, Portugal, 2001.

[19] R. Habachi, A. Touil, A. Charkaoui, and A. Echchatbi, "Eagle strategy based crow search algorithm for solving unit commitment problem in smart grid system," Indonesian Journal of Electrical Engineering and Computer Science., vol. 12, no. 1, pp. 17-29, 2018.

[20] S. S. Sakthi, R. K. Santhi, N. Murali Krishnan, S. Ganesan, and S. Subramanian, "Wind Integrated Thermal Unit Commitment Solution Using Grey Wolf Optimizer," International Journal of Electrical and Computer Engineering (IJECE), vol. 7, no. 5, p. 2309, Oct. 2017.

[21] J. Kennedy, R. Eberhart, "Particle swarm optimization," in: IEEE International Conference on Neural Networks, Vol. 4, 1995, pp. 1942-1948.

[22] R. Eberhart, J. Kennedy, "A new optimizer using particle swarm theory," in: IEEE Proceedings of the Sixth International Symposium on Micro Machine and Human Science, 1995, pp. 39-43.

[23] J. Kennedy and R. C. Eberhart, "A discrete binary version of the particle swarm algorithm," IEEE International Conference on Systems, Man, and Cybernetics. Computational Cybernetics and Simulation, Orlando, FL, USA, 1997, pp. 4104-4108 vol.5.

[24] Han K.H and Kim J.H, "Quantum-inspired evolutionary algorithm for a class of combinatorial optimization," in IEEE Transactions on Evolutionary Computation, vol. 6, no. 6, pp. 580-593, Dec. 2002.

[25] L. Spector, et al., "Finding a better-than-classical quantum AND/OR algorithm using genetic programming," Proceedings of the 1999 Congress on Evolutionary Computation-CEC99 (Cat. No. 99TH8406), Washington, DC, USA, 1999, pp. 2239-2246 Vol. 3.

[26] Y. Jeong, et al., "A New Quantum-Inspired Binary PSO: Application to Unit Commitment Problems for Power Systems, ” in IEEE Transactions on Power Systems, vol. 25, no. 3, pp. 1486-1495, Aug. 2010. 


\section{BIOGRAPHIES OF AUTHORS}

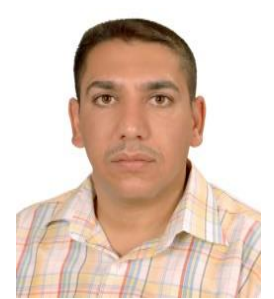

Ali Nasser Hussain was born in Iraq on April 30, 1974. He received his B.Sc. and M.Sc. in Electrical \& Electronics Engineering, University of Technology, Baghdad, Iraq, in 1998 and in 2005 respectively and his PhD degrees in Electrical Engineering from University Malaysia Perlis (UniMAP), Perlis, Malaysia in 2014. Since 2004 he is a senior lecturer in the Electrical Engineering Technical College at Middle Technical University. His current research interests include power system operation and control, electrical power system stability and intelligent optimization, renewable energy, robust control.

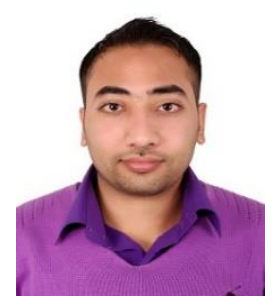

Ali Abduladheem Ismail was born in Baghdad-Iraq on February 17, 1989. He received his B.Sc. in Electrical Power Engineering Techniques, Electrical Engineering Technical College, Middle Technical University, Baghdad, Iraq, in 2010.He is a shift engineer in National Dispatch Control Center (NDCC), Ministry of electricity, Iraq. He is pursuing the M.Sc. degree at Electrical Engineering Technical College, Middle Technical University. His current research interest includes optimal operation of power system and economics. 